\title{
Enostosis Mimicking a Supernumerary Tooth- A Case Report
}

\section{Srikrishna Vempaty*}

Southend University Hospital NHS Foundation Trust, Essex SSO ORY, United Kingdom

\begin{abstract}
An incidental finding of radiopaque shadow on extraoral orthopantomogram in mandible interpreted as a supernumerary tooth and referred for extraction. Cone beam computer tomography scan confirmed it to be a benign focus of mature compact bone which is diagnosed as enostosis. Three dimensional imaging in this case proved to be vital to reach diagnosis. The diagnosis of enostosis and its implications to orthodontic movement is a subject of interest. We advised to orthodontist to reconsider his plan and make changes.
\end{abstract}

Keywords: Orthopantamogram; Cone beam computer tomography; Radiopaque lesion; Exostosis; Mandible

\section{Introduction}

Radiopaque lesions are a common radiological incidental finding. Some of them cause dilemma as they can be asymptomatic and cannot be correlated with complaints of the patient. The aim of this case report is to address the value of Cone Beam Computer Tomography (CBCT) scan as second line imaging modality in investigating asymptomatic radiopacities of clinical significance. This case proves that CBCT scan following an extraoral orthopantomogram helped in determining the extent of lesion and its clinical significance [1]. The other alternative investigation would be a conventional multidirectional computer tomography or low dose technique computer tomography scan [2].

\section{Case Presentation}

A 17 year old female with history of malaligned teeth was referred from orthodontist for extraction of a supernumerary tooth along with first premolars in lower jaw. This is a usual practice to gain space for future orthodontic treatment. The referral included an orthopantomogram revealing a dense, well defined radiopaque lesion in relation to the periapical region of lower left first and second premolars (Figure 1). It is horizontal in alignment measuring $2 \mathrm{~cm}$ in length and $5 \mathrm{~mm}$ in width lying parallel to lower border in close proximity with roots of premolars. On examination, clinically there was no cortical bone expansion or enlargement on the labial or lingual side. The patient did not show any signs or symptoms of infection. The orthodontist considered this radiopaque lesion (supernumerary tooth in jaw) to interfere with his orthodontic treatment or potentially cause resorption of roots.

In view of determining the proximity of this radiopacity to surrounding structures, we undertook a CBCT scan of the mandible, which ruled out the presence of a supernumerary tooth. The CBCT also confirmed it to be cortical bone with increasing density towards the medulla. This matches the description of enostosis to say that it is a focus of mature compact (cortical) bone within the cancellous bone (spongiosa) [3].

The enostosis was not involving the teeth in proximity or their supporting structures. These finding rules out the possibility of Cemento ossifying dysplasia, where the lesions develop from the undifferentiated cells of periodontal ligament tissues [4,5]. CBCT also confirmed that there were no other radiopaque lesions in the mandible which is a common finding in florid cemento osseous dysplasia (Figure 2) [6].

\section{Discussion}

Following the CBCT scan, the orthodontist was informed about the diagnosis of enostosis and suggested altering the treatment plan accordingly. Although this is a relatively common benign bone condition, the orthodontic implications in realigning teeth across an area of enostosis are worth investigating. Orthodontic movement

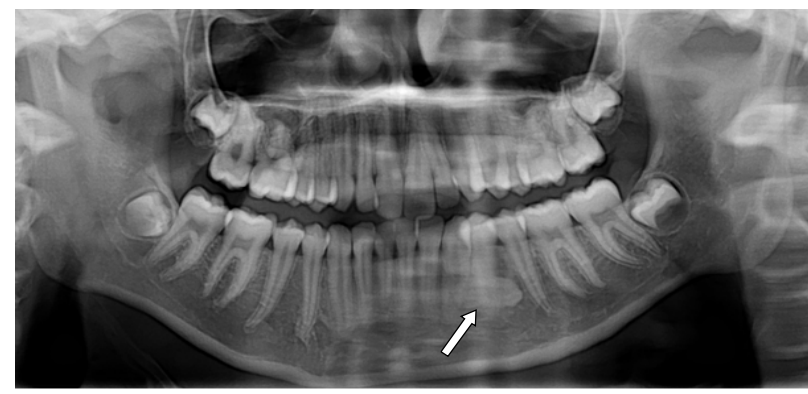

Figure 1: Radiopaque lesion (Arrow) extends from roots of lower left canine to lower left second premolar lying parallel to lower border of mandible.

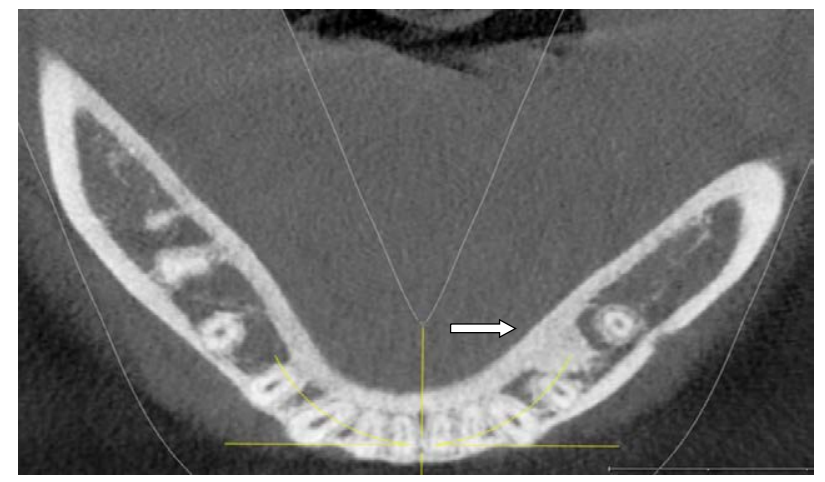

Figure 2: Axial view of Cone beam computer tomogram (СBCT) showing radiopaque focus of mature compact bone extending into cancellous bone on left premolar region.

*Corresponding author: Srikrishna Vempaty, Speciality Doctor Oral and maxillofacial surgery, Southend University Hospital NHS Foundation Trust, Essex SS0 0RY, United Kingdom, Tel: +447939655893; E-mail: skrisv76@hotmail.com

Received May 01, 2015; Accepted June 02, 2015; Published June 04, 2015

Citation: Vempaty S (2015) Enostosis Mimicking a Supernumerary Tooth- A Case Report. Dentistry 5: 310. doi:10.4172/2161-1122.1000310

Copyright: @ 2015 Vempaty S. This is an open-access article distributed under the terms of the Creative Commons Attribution License, which permits unrestricted use, distribution, and reproduction in any medium, provided the original author and source are credited. 
through a dense bone could potentially increase the risk of root resorption and loss of tooth vitality due to excessive force [7]. There has been no consensus on this subject [8]. Literature on similar lesions advises regular monitoring of the lesions by clinicians [8].

\section{References}

1. Araki M, Hashimoto K, Kawashima S, Matsumoto K, Akiyama Y (2006) Radiographic features of enostosis determined with limited cone-beam computed tomography in comparison with rotational panoramic radiography. Oral radiology 22: 27-33.

2. Jeong DK, Lee SC, Huh KH, Yi WJ, Heo MS, et al. (2012) Comparison of effective dose for imaging of mandible between multi-detector CT and conebeam CT. Imaging Sci Dent 42 : 65-70.

3. Greenspan A (1995) Bone island (enostosis): current concept-a review. Skeletal Radiol 24: 111-115.
4. Eskandarloo A, Yousefi $F$ (2013) СBCT findings of periapial cemental dysplasia- A case report. Imaging Sci Dent 43: 215-218.

5. Alsufyani NA, Lam EM (2011) Cemento-osseous dysplasia of jaw bones: key radiographic features. Dentomaxillofac Radiol 40: 141-146.

6. Mac Donald-Jankowski DS (2003) Florid cement-osseous dysplasia: a systemic review. Dentomaxillofacial Radiol 32: 141-149.

7. Marques-Silva L, Guimarães ALS, Dilascio MLC, Castro WH, Gomez RS (2007) A rare complication of idiopathic osteosclerosis. Med Oral Patol Oral Cir Bucal 12: E233-234.

8. Nakano K, Ogawa T, Sobue S, Ooshima T (2002) Dense Bone Island: clinica features and possible complications. Int J Paediatr Dent 12: 433-437. 